of rheumatoid arthritis (RA). Earlier we showed that already at the seropositive "at risk" stage uninflamed synovial tissue contains T-cell infiltrates ${ }^{1}$. In another study we showed that inflamed synovium selectively harbours expanded T-cell clones that are hardly present in paired blood samples ${ }^{2}$.

Objectives: Following up on these observations, we longitudinally investigated whether the same expanded T-cell clones found in the inflamed synovial tissue at onset of RA are already present in the synovium in the seropositive "at risk" stage.

Methods: Fifty-five individuals without arthritis but seropositive for IgM rheumatoid factor and/or anti-citrullinated protein antibody (ACPA) were prospectively followed. In five aCCP+ individuals synovial biopsies and paired blood samples at inclusion ("at risk" stage) and after development of RA (ACR2010 criteria; mean time to arthritis 27 months (range 11.7-47.3)) were available for analysis. T-cell clones were identified by their unique TCR $\beta$ sequence using RNA-based next generation sequencing $^{3}$. For each sample, 3570 TCR $\beta$ sequences were analysed. Clones with a frequency of $\geq 0.5 \%$ were arbitrarily considered as highly expanded clones (HECs). ANOVA and t-test were used for statistical analysis.

Results: T-cell repertoires in "at risk" and RA synovium were similar (mean ( \pm SD) number of clones $488 \pm 70$ vs $567 \pm 204$ respectively, $p=0.46$ ), number of HECs $(37 \pm 7$ vs $31 \pm 18, p=0.41)$ and the impact of HECs collectively on the TCR repertoire (mean $48 \% \pm 13 \%$ vs $50 \% \pm 20 \%, \mathrm{p}=0.84$ ). Interestingly, of the HECs present in the synovium at onset of arthritis $23 \%( \pm 9 \%)$ were already present as HECs in the synovium at the seropositive "at risk" stage. This overlap was significantly higher than that with paired blood samples taken at the arthritis $(3 \%$ $\pm 3 \% ; p=0.01)$ or at the seropositive "at risk" stage $(5 \% \pm 7 \% ; p=0.01$; Figure 1a-c patient example; Figure 1d summary of results). Further characterization of the synovial CDR3 sequences (length, total charge, polar, aromatic and aliphatic side chains) showed no significant differences between RA HECs that were and those that were not expanded in the seropositive "at risk" stage.

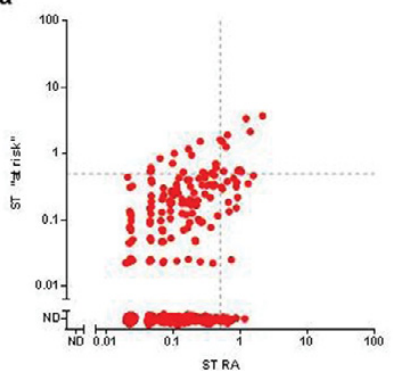

c

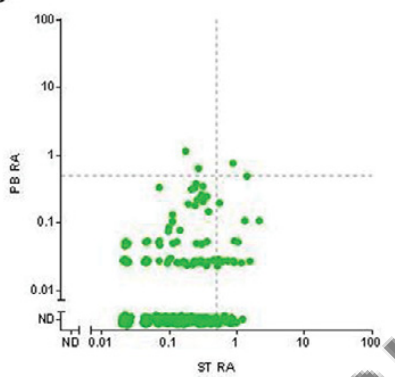

b

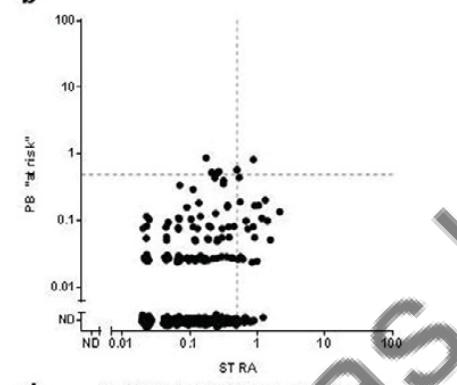

d

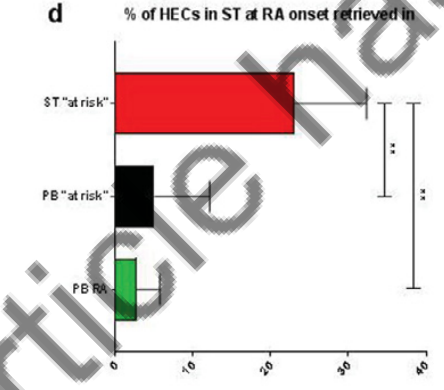

Figure 1: (a-c) Axes show the frequency of the $\mathrm{XCR}$ dones in the depicted samples for one patient. $\mathrm{ND}=$ non-detected. (d) Summary of results from 5 spatients, ${ }^{* *} \mathrm{p}=0.01$

Conclusions: Many T-cell clones found in early RA synovial tissue are already present in the pre-clinical "at risk" phase. The resemblance in TCR repertoires indicates that the process leading to disease - at least at the T-cell level - constitutes a smooth development. These clones, being already present in the very early stage of this disease and persisting as dominant clones during contraction of active arthritis, form attractive candidates for further characterization. References:

[1] de Hair MJ et al. Arthritis Rheum. 2014.

[2] Klarenbeek PL et al. Ann Rheum Dis. 2012.

[3] Klarenbeek PL et al. Immunol Lett. 2010.

Disclosure of Interest: None declared

DOI: 10.1136/annrheumdis-2017-eular.6318

\section{OP0173 DOMINANT B-CELL RECEPTOR CLONES IN PERIPHERAL BLOOD PREDICT ONSET OF ARTHRITIS IN INDIVIDUALS AT RISK FOR RHEUMATOID ARTHRITIS}

P.-P. Tak ${ }^{1}$, M.E. Doorenspleet ${ }^{2,3}$, M. de Hair ${ }^{4}$, P. Klarenbeek ${ }^{2,3}$, M. van Beers-Tas ${ }^{5}$, A. van Kampen ${ }^{6}$, D. van Schaardenburg ${ }^{5}$, D. Gerlag ${ }^{4}$, F. Baas ${ }^{3}$, N. de Vries ${ }^{2} .{ }^{1}$ Department of Clinical Immunology and Rheumatology;

${ }^{2}$ Amsterdam Rheumatology and Immunology Center; ${ }^{3}$ Department of Genome Analysis, ${ }^{4}$ Clinical Immunology and Rheumatology, Academic Medical Center;
${ }^{5}$ Amsterdam Rheumatology and Immunology Center, Reade; ${ }^{6}$ Department of Clinical Epidemiology, Biostatistics and Bioinformatics, Academic Medical Center, Amsterdam, Netherlands

Background: The onset of seropositive rheumatoid arthritis (RA) is preceded by the presence of specific autoantibodies in the absence of synovial inflammation. Only a subset of these at-risk individuals will develop clinical disease. This impedes efforts to implement early interventions that may prevent onset of clinical disease.

Objectives: Here we analyze whether clonal changes in the B-cell receptor (BCR) repertoire can reliably predict onset of clinical disease.

Methods: In a prospective cohort study in 21 individuals at-risk for RA, the BCR repertoire of paired peripheral blood and synovial tissue samples was analyzed using next-generation BCR sequencing. BCR clones that were expanded beyond $0.5 \%$ of the total repertoire were labeled dominant. The relative risk for onset of arthritis was assessed, using a cut-off of presence of $\geq 5$ dominant BCR clones. Findings in peripheral blood were validated in an independent prospective cohort of 50 at-risk individuals. Based on the test cohort, individuals in the validation cohort were considered positive if peripheral blood at study entry showed $\geq 5$ dominant BCR clones.

Results: Both in the test and validation cohort, the presence of $\geq 5$ dominant BCR clones in peripheral blood was significantly associated with arthritis development (validation cohort relative risk (RR) 6.3, 95\% confidence interval $(\mathrm{Cl}) 2.7-15$, $\left.\mathrm{p}<1^{*} 10^{-4}\right)$. Even when adjusted for the recently described clinical prediction rule the association remained intact (relative risk $5.0,95 \% \mathrm{Cl} 1.2-20, \mathrm{p}=0.024$ ). When individuals developed arthritis, dominant BCR clones disappeared from peripheral blood and appeared insynovial tissue, suggesting a direct role of these clones in disease pathogenesis.

Conclusions: Dominant BCR clones in peripheral blood predict onset of clinical symptoms of RA in at-risk individuals with high accuracy. Our data suggest that during onset of RA these clones shift from peripheral blood to target tissue.

Disclosure of Interest: P.-P. Tak Employee of: GlaxoSmithKline, Stevenage, United Kingdom, M. Doorenspleet: None declared, M. de Hair: None declared, P. Klarenbeek: None declared, M. van Beers-Tas: None declared, A. van Kampen: None declared, D. van Schaardenburg: None declared, D. Gerlag Employee of: Clinical Unit Cambridge, GlaxoSmithKline, Cambridge, United Kingdom, F. Baas: None declared, N. de Vries: None declared

DOI: 10.1136/annrheumdis-2017-eular.4323

.

OP0174 NEW PROTEIN ARRAY TECHNOLOGY IDENTIFIES RITUXIMAB TREATED NON RESPONDER RHEUMATOID ARTHRITIS PATIENTS ARE GENERATING A NEW AUTOANTIBODY REPERTOIRE

Z. Konthur ${ }^{1}$, M. Wiemkes ${ }^{2}$, T. Häupl ${ }^{2}$, G. Burmester ${ }^{1}$, K. Skriner ${ }^{2}$. ${ }^{1}$ Max Planck Institute, Max Planck Institute; ${ }^{2}$ Department of Rheumatology and Clinical Immunology, Charité University Medicine, Berlin, Germany

Objectives: Rituximab (RTX) has shown clinical efficacy but up to $40 \%$ of RTX treated rheumatoid arthritis (RA) patients are poor responders (Ann-Rheum-Dis. 2005 Feb;64(2):246-52) and the commonly used RA biomarkers (RF/ACPA) are poor predictors for therapy response. In this study the autoantibody repertoire analysed on protein macorarrays from RA patients under RTX treatment was correlated to clinical DAS28 response.

Methods: Screening of RA sera was conducted on 37.830 unique human proteins on protein marcoarrays (http://www.engine-gmbh.de) with sera taken before and 24 weeks after treatment. The autoantibody response of different immunoglobulin classes $\lg \mathrm{D}, \lg \mathrm{A}$, and $\lg \mathrm{G}$ was recorded and bioinformatically evaluated. Response was determined according to DAS28 criteria. DAS 28 scores in the responder group before treatment was from $5.4-7.8$ and in the non-responder group $5,6-6,8$. We analyzed 26 RA patient sera (9 responder, 7 non-responder and 10 patients with blinded response classification) investigated the data of found autoantigens in-silico and by hierarchical clustering

Results: In the cohort of 26 patients 1292 different autoantigens $(\lg D, \lg A, \lg G)$ were detected. Using protein array we investigated clusters of autoantigen responses that disappeared or developed during RTX treatment of RA patients. RA autoantigenic patterns before and 6 month after RTX treatment were patientspecific and no relevant autoantigenic cluster was found that was shared between patients or associated with response. However, RTX reduced the repertoire of autoantibodies after 24 weeks of treatment in the tested RA patient cohort on average by $60 \%$. RA patients which do not respond are generating on average $63 \%$ new autoantibodies. In good responders to RTX only $5,5 \%(+/-3 \%)$ new autoantibodies can be detected. The $\lg A$ and $\lg G$ autoantibody repertoire in the serum after 24 weeks of RTX treatment is reduced (IgA: $41 \%$, IgG: $31 \%$ ) in good responders whereas it is increased ( $\lg A: 1,3 \%, \lg G: 24 \%)$ in non responders to RTX.

Conclusions: After 6 month of RTX treatment the autoantibody repertoire in all good responding RA patients is reduced and non responders to RTX change their autoantibody repertoire directed against new but patient specific antigens. The fast rebuilding of functional B cells is only detected in non-responders to rituximab.

Disclosure of Interest: None declared

DOI: 10.1136/annrheumdis-2017-eular.6978 